\title{
Decoherence and Intertheory Relations in Quantum Realism*
}

Decoherencia y relaciones interteóricas en el realismo cuántico

Nahuel Sznajderhaus ${ }^{\dagger}$

\begin{abstract}
The complex relation between quantum mechanics and classical mechanics is crucial in the philosophy of modern physics, and it cuts across current quantum physics. This paper is divided in two parts. In the first part I will offer a critical analysis of the role that decoherence plays in the account of the quantum-classical limit. In the second part I will mention three ways in which philosophers are engaging with the realist interpretation of quantum mechanics in light of the assessment that the problem of the quantum-classical limit is still open to debate. My main claim is that the problem of the quantum-classical limit is overrated and it receives too much attention for the realist who looks at quantum mechanics. The question that the realist wants to focus on is the crucial interpretation question: what is a quantum system?
\end{abstract}

Keywords: realism - quantum mechanics - decoherence - quantum-classical limit

\section{Resumen}

La compleja relación entre la mecánica cuántica y la mecánica clásica es de crucial importancia para la filosofía de la física de hoy. Este artículo está dividido en dos partes. En la primera parte se ofrece un análisis crítico sobre el rol que la decoherencia juega en la concepción estándar del llamado límite cuántico-clásico. En la segunda parte se presentan tres maneras distintas de tomar una postura frente al problema de interpretación de la mecánica cuántica en función de la observación de que la descripción del problema del límite cuántico-clásico está aún irresuelto. El enunciado principal que se defiende en este artículo es la importancia del problema del límite cuántico-clásico dentro del desafío de la interpretación realista de la mecánica cuántica está sobreestimada. De hecho, el límite cuántico-clásico distrae al realista de la pregunta fundamental: ¿qué es un sistema cuántico?

Palabras clave: realismo - mecánica cuántica - decoherencia - límite cuántico-clásico

\footnotetext{
* Received: 7 June 2018. Accepted in revised version: 8 September 2018.

${ }^{\dagger}$ National University of Ireland, Ireland. To contact the author, please write to: nsznaj@gmail.com. Metatheoria 9(2)(2019): 95-110. ISSN 1853-2322. eISSN 1853-2330.

(c) Editorial de la Universidad Nacional de Tres de Febrero. Publicado en la República Argentina.
} 
"A theory that is merely logically consistent and gives accurate predictions is not yet a good theory. We want much more: we want to see something never seen before" (Primas 1981, p. 22).

\section{Introduction}

The realist interpretation of quantum mechanics remains a major challenge in current philosophy of science. Typically, a debate on this issue will focus on defending or attacking one of the many interpretations such as many worlds interpretations, the spontaneous collapse interpretation developed initially by Ghirardi Rimini and Weber (GRW), Bohmian interpretations. Despite the existing variety of interpretations, there is no consensus of which, if any, is the correct one. This paper takes seriously that the substantial disagreement on the interpretation of quantum mechanics indicates that the realist questions about the realist content of the theory have currently no definite answer.

In this paper I will consider the general problem of the account of the intertheory relation quantum-classical. Indeed, significant developments in physics are stimulated by the account of the appearance of the classical from the quantum, and well-known theoretical devices have been articulated in physics to account for such a relation: mathematical limits, Ehrenfest theorem, Wigner function and, perhaps more importantly, decoherence. These were, to a large extent, specifically designed to account for the relationship between quantum mechanics and classical mechanics.

One might take a historical viewpoint and consider that the development of quantum mechanics initially intended to mean a replacement of classical mechanics (the well-known ultraviolet catastrophe and the subsequent quantum account of it, for instance). However, the subsequent development of the quantum theory after the initial breakthroughs in the early 1910s made it evident that quantum mechanics departed significantly from classical mechanics. The mature theory obtained by the 1930s was something else than a replacement of classical mechanics. Despite the difficulty of entirely replacing classical mechanics, the broad expectation that quantum mechanics should show the appearance of classical mechanics remained. This can be conceptualised in the philosophical tradition by theory reduction, which is considered the standard account of the way that theories relate with each other. ${ }^{1}$

This article is divided in two parts. In sections 2 and 3 I offer a critical analysis of the status of decoherence within the broader issue of the account of the quantum-classical limit. I will argue that decoherence is a theoretical device that was designed by physicists in order to resolve the issue of intertheory relation quantum-classical. In Section $4 \mathrm{I}$ offer an analysis of the kind of reactions that relevant authors have had in the face of the generally unsatisfactory account of the issue of intertheory relations. I conclude by offering a novel strategy that swings the realist back to the basic interpretation question: what is a quantum system? Or, what is the world like according to quantum mechanics? This is the fundamental issue that the realist should solve, for how to account for the relation between two theories when we still do not know what the realist content of one of them is?

\section{The received account of the quantum-classical limit and its problems}

A central issue in philosophy of science focuses on accounting for how different scientific disciplines and theories relate with each other. Indeed, in the philosophy of physics, relevant research has been and still is being undertaken with this type of question at the centre (e.g. Bokulich 2008a, Fletcher 2014, Rosaler 2016). As Butterfield indicates, there are two plausible broadly construed intuitions of

\footnotetext{
1 The traditional view is theory reduction, although there are other ways in which one could consider quantum mechanics to be related to classical mechanics without reduction, for instance emergence. What views like these have in common is the vertical hierarchy of fundamentality: quantum mechanics is more fundamental than classical mechanics.
} 
reduction or pluralism, ${ }^{2}$ both equally capable of being developed in epistemic terms (e.g. explanation) or in metaphysical terms (e.g. identity of entities or properties):

One of the tasks of philosophy of science is to assess how well integrated our theories are. Indeed: are they integrated enough in terms of notions like explanation and the identity of theoretical entities or properties, that taken together they merit the metaphor 'raising edifice', rather than 'shambolic patchwork?! (Butterfield 2011, p. 930)

A widely accepted view tells us that psychology "reduces to" or "emerges from" neurosciences, that the latter is similarly related to biology, which then can be related to chemistry, and so on until one recognises that all sciences can be thus recovered from fundamental physics. ${ }^{3}$ A similar view is also taken within physics itself. Particular cases in intertheory relations consider, for instance, the reduction between special relativity and Newtonian mechanics $(\mathrm{CM}){ }^{4}$ This is essentially based on the mathematical fact that the Galilean transformations tend to Lorentz transformations when the ratio $\frac{v^{2}}{c^{2}} \rightarrow 0$. Another example-yet not uncontroversially accepted across the board-is the relationship between classical statistical mechanics and thermodynamics: from considerations of the relevant casedependent type of ensemble, one can recover laws of thermodynamics in terms of average values of statistical functions.

Now, the case I will focus on is the intertheory relation between quantum mechanics and classical mechanics. There are well-known theoretical devices that attempt to account for how classical mechanics appears from quantum mechanics: mathematical limits, Ehrenfest theorem, Wigner function and, perhaps more importantly, decoherence. It is agreed that all of these work only in a limited number of cases, and decoherence is the most relevant and prone to philosophical engagement. Indeed, this article critically engages with the crucial role and relevance of decoherence in the account of the realist interpretation of quantum mechanics. In the next section I will exhibit existing disagreement on what decoherence is. In Section 4 I will enumerate three alternatives to the considerations of the role that decoherence plays within the broader picture of the interpretation of quantum mechanics. By the end I will argue for the possibility of a fourth alternative.

\section{The many faces of decoherence}

In this section I discuss the significance of decoherence in the problem of the quantum-classical limit. I defend that decoherence was created with the aim of providing an account of the appearance of the classical from the quantum, and I will do so by looking at the views of the physicists that created the standard models of decoherence. However, it is also the case that relevant authors in the foundations of physics disagree on how to conceive the quantum-classical limit itself and the role that decoherence plays within it.

Decoherence was developed by challenging the notion that quantum systems are closed and isolated from their environment (Zeh 1970). Of course, all systems interact with their environment, but the qualitative step in the appreciation of decoherence is that the interaction between the system and its environment is such that the system cannot even be considered as approximately isolated. Hence, 'decoherence' is the name for the quick 'disappearance' of the coherence in the superposition terms across macroscopically different properties, due to the interaction between the system and the environment (Joos 1996, p. 2).

\footnotetext{
${ }^{2}$ Given that I consider that theory reduction is much closer to the practice of physicists than pluralism, in this article I will focus on the former. By contrast, Bokulich (2008a) views it that the mainstream view on intertheory relations falls within pluralism, but I am not convinced by her arguments and addressing this will not concern me here.

${ }^{3}$ I understand this type of comment might trigger questions from other disciplines in philosophy, such as the question whether mental states are physical or not, and the like. I do not intend to engage in those discussions here, as will become clear soon.

${ }^{4}$ For my purposes I will overlook the nuances of Newtonian mechanics in relation to the currently accepted versions of classical mechanics. I will consider the different variants of classical mechanics all the same.
} 
Evidenced by the immense written literature, technological progress and funding invested, decoherence plays a central role in frontier projects. Zurek (2003) lists a large amount of applications and consequences of decoherence in physics, see also (Cirac \& Zoller 1995). Environment-induced decoherence goes through much of the cutting-edge physics, both in theoretical and experimental areas. Nevertheless, much of the physics literature does not make concerted efforts to engage with philosophical reflection on physics, and thus the philosopher of science has valuable contributions to make.

However pragmatic the role of decoherence in the practice of physics might be, there are some considerations relevant to the philosopher-and to the realist in particular-that should be taken with extreme caution. While there is no disagreement on the remarkable instrumental value of decoherence, there is substantial disagreement on its status and its role in the realist account of quantum mechanics, as I will discuss. Indeed, against the view of some physicists and philosophers, I defend that decoherence does play a crucial role in accounting for the quantum-classical limit, and that it was conceived within the task of showing the appearance of the classical from the quantum.

Let us discuss some significant vagueness in the conception of decoherence in the literature on foundations of quantum mechanics. Zeh (1996), one of the founding fathers of decoherence, talks about the programme of decoherence, the theory of decoherence, the phenomenon of decoherence, and also in these terms: "Decoherence by 'continuous measurement' (as it was originally called) seems to represent the most fundamental irreversible process in Nature" (Zeh 1996, p. 12, my emphasis). In turn, Joos (1996, p. 2) talks about the mechanisms of decoherence. Finally, Schlosshauer (2007, p. viii) mentions decoherence as a programme. I do not argue that these terms entail an outright contradiction, but I do claim that this is not mere verbal disagreement. Whilst for the physicists all these terms might sound unproblematically similar, the philosopher is capable of recognising substantial differences. ${ }^{5}$

Now let me argue that decoherence does play a role in the account of the quantum-classical limit, being something more than 'just a quantum phenomenon that occurs whenever the system interacts with its environment'. For instance, Zurek (2003, p. 717) expresses that decoherence provides the account of "how the environment distills the classical essence from quantum systems", an account of "why the quantum universe appears classical when it is seen 'from within'” (Zurek 2003, p. 718); in turn, Joos opens the introduction to the collection of works about decoherence in the following way: "What distinguishes classical from quantum objects? What is the precise structure of the transition from quantum to classical? Is this transition smooth and harmless, or does it rather involve a sudden, abrupt change of concepts?" (Joos 1996, p. 1); further, in that same collection titled "Decoherence and the Appearance of a Classical World in Quantum Theory", Zeh declares that "the theory of decoherence is to explain the difference in appearance between the quantum and the classical under the assumption of a universally valid quantum theory" (Zeh 1996, pp. 8-9); finally, in his comprehensive review of the account of the quantum-classical limit, Landsman explains that "originally, decoherence entered the scene as a proposed solution to the measurement problem and its goal is to explain the approximate appearance of the classical world from quantum mechanics seen as a universally valid theory" (Landsman 2007, pp. 515-517). I hope that it will be clear to the reader that these comments support my claim that decoherence plays an explicit and relevant role in the account of the quantum-classical limit.

Therefore, I think it can be argued that decoherence is an account of the quantum-classical limit, or the explanation of the appearance of classicalities from the quantum, or the justification for why the world appears classical, given that it is not. Yet, there are some useful clarifications to make before we go any further. It should be recognised that part of the physics community that perhaps does not engage in much philosophical reflection considers decoherence to be a phenomenon that occurs in nature whenever a system interacts with the environment. However, as I will justify in detail, decoherence can also be seen as a theoretical device that attempts to account for the quantum-classical

\footnotetext{
${ }^{5}$ I am grateful to Paul Knott for pressing on this point.
} 
limit. And I will motivate this assessment by looking at relevant physicists and philosophers of physics who work in foundations of quantum mechanics, such as Zeh, Zurek, Joos, Bacciagaluppi, Landsman, Schlosshauer. Indeed, there is currently a hot debate on how to conceive the quantum-classical limit itself and the role that decoherence plays within it. Now, I do not intend to engage with all the relevant views. Instead, I just want to show sufficient disagreement on the terms and conditions of the problem. For example, Bacciagaluppi (2013) considers the measurement problem as a separate problem to the quantum-classical limit (in the sense of the limit between the classical regime and the regime of quantum mechanics), and argues that the solution to one problem does not solve the other. Bacciagaluppi argues that decoherence does not solve the measurement problem and that decoherence recovers predictions of classical mechanics merely instrumentally. That is, he considers that a strong realist interpretative programme is still needed. Therefore, he concludes, the problems remain open to further discussion.

By contrast with Bacciagaluppi, Schlosshauer (2007, p. 49ff.) considers that the quantum-classical limit is a broader problem that has the measurement problem as a component of it. Furthermore, that the latter is actually three problems: the problem of the preferred basis, the problem of explaining the non-observability of superpositions in the macroscopic scale, and the problem of the outcomes. His assessment is that decoherence solves the first two, but does not provide an answer to why there is an outcome to the measurement process.

To show yet another view, Landsman $(2007$, p. 419) presents the mathematical limits-e.g. $t \rightarrow 0$ and $n \rightarrow \infty$, which involve taking limits of equations, the Ehrenfest theorem, and decoherence, as the devices that intend to solve the problem of explaining the appearance of the classical world from quantum theory. His assessment is that all of them ultimately fail, strictly speaking. The important point to emphasise is that Landsman considers that these theoretical devices (including decoherence) are mainly there to account for the appearance of the classical world given that it is quantum.

With this I showed various views of presenting what precisely decoherence is, and what role it plays in the interpretation of quantum mechanics. And the realist that recognises that decoherence is meant to show how the classical appears from the quantum, can then acknowledge that such is a claim of intertheory relations. There is disagreement on phrasing the problem, there is also disagreement on whether decoherence solves the problems or not, but where there is agreement is that decoherence is meant to address the relationship between the quantum and the classical. Yet, it is known that there is disagreement on the realist interpretation of quantum mechanics itself, so how could we possibly engage with establishing the relation between two theories, one of which has still no clear realist interpretation-i.e. quantum mechanics?

Therefore, decoherence plays a role in the problem of the quantum-classical limit understood the measurement problem and the preferred basis problem, see Schlosshauer (2005). I will agree with Landsman (2007) and Bacciagaluppi $(2013,2016)$, in that decoherence ultimately fails to solve these problems (contra Schlosshauer). ${ }^{6}$

Now, say that you have achieved describing a smooth and uncontroversial transition from quantum mechanics to classical mechanics via some formal device like decoherence. And then say that you infer from such a smooth transition that there is a metaphysical connection between quantum objects and classical ones. Then, that still does not remove the need for the question of the very nature of the quantum objects, for we assumed that there is no clear solution to the question for the realist interpretation of quantum mechanics. Hence, would it not be methodologically unsound to attempt to address a relationship between quantum mechanics and classical mechanics, given that the realist has still not managed to spell out the nature of quantum objects any further than claiming that they are what quantum states represent, or that they are whatever the physicists use to make predictions?

\footnotetext{
${ }^{6}$ For space reasons, I will assume that the reader is familiar with the measurement problem and the preferred basis problem. For useful discussions see Zurek (1981) and Schlosshauer (2005, 2007).

7 Hence, for example many worlds interpretation is currently considered by Wallace (2016) to be a theory of the decoherent macro-world. And, when asked about the micro-world, Wallace would reply that there is just a coherent quantum state. This response seems to address a quantum-classical limit, explaining the appearance of the macro-world. However, it spells out the physical content of quantum
} 
I argue that the two questions-the intertheory relation between quantum mechanics and classical mechanics, and the interpretation of quantum mechanics-ought to come at significantly different stages in the development of a philosophical view on physics. A sound methodology would first have a conceptual apparatus, or, in the terms of French and McKenzie (2012), the right 'metaphysical tool' in order to interpret the formalism of quantum mechanics and be able to realistically account for and explain the experimental results. ${ }^{8}$ At a second stage, such a sound methodology would be able to explore the possible relationship between quantum mechanics and classical mechanics. By contrast, the very essence of the currently dominant view on physics seems to be the contrary to the methodology I just described. If this makes some sense and decoherence plays a part in the broader issue of addressing the relation quantum-classical, then this would indicate that a large part of today's literature focuses on a problem of intertheory relation, rather than on the central issue in the interpretation of quantum mechanics. This is a main claim in this paper: that the interpretation of quantum mechanics is today merging two a priori different issues: the account of intertheory relations, and the interpretation of the theory.

In order to show and then critically engage with how decoherence works, I will quickly discuss the well-known model of the 'decoherence of a single qubit' taken from Zurek (1982, pp. 1864ff.), Zurek (2003, pp. 730ff.) and d'Espagnat (1995, Sect. 10.6).

\subsection{A standard model of decoherence}

Let us recall a simple model of decoherence. Decoherence is discussed widely in the literature in various guises, yet I will follow the seminal paper (Zurek 1982), which is still valid and a primary source for this topic. Indeed, Schlosshauer (2005, Sect. III.D.2) is essentially identical to Zurek's 1982 paper.

Consider a $1 / 2$-spin particle as our system $S$ described in the $z$ direction with the kets $|\uparrow\rangle$ and $|\downarrow\rangle$ and the apparatus $A$ also a two-state system-described by the states $\left\{\left|p_{+}\right\rangle,\left|p_{-}\right\rangle\right\}$. As Zurek (1982, p. 1864) explains, the apparatus can be seen as an atom with a ground and excited states with the same energy, which can be formally considered as another $1 / 2$-spin system. The self-energies of the apparatus and the system can be ignored, and only an interaction hamiltonian $H^{S A}$ is considered. $\mathrm{H}^{\mathrm{SA}}$ operates for a short time and its intensity is determined by $g$, a coupling constant

$$
\mathrm{H}^{\mathrm{SA}}=\mathrm{g}(|\uparrow\rangle\langle\uparrow|-| \downarrow\rangle\langle\downarrow|) \otimes\left|\mathrm{p}_{+}^{z}\right\rangle\left\langle\mathrm{p}_{+}^{z}|-| \mathrm{p}_{-}^{z}\right\rangle\left\langle\mathrm{p}_{-}^{z}\right| .
$$

In this situation, $\mid p_{\mp}^{Z}>$ are the two possible outcomes of the apparatus that are correlated to the spin $\mathrm{s}^{2}=\mp 1$ of the system $S$ (in units of $\hbar / 2$ ). The initial state for the system $S$ is a linear combination of the basis states in $z$ :

$$
\left|\phi_{\mathrm{i}}\right\rangle=\mathrm{a}|\uparrow\rangle+\mathrm{b}|\downarrow\rangle,(2)
$$

with $a, b \in C$ and $|a|^{2}+|b|^{2}=1$, and consider $\left|p_{+}^{x}\right\rangle$ the initial state of the apparatus $A$. The hamiltonian in eq. (1) will evolve the system as follows:

$$
\left|\Psi_{\mathrm{i}}\right\rangle=\left|\phi_{\mathrm{i}}\right\rangle \otimes\left|\mathrm{p}_{+}^{\mathrm{x}}\right\rangle \rightarrow \mathrm{a}|\uparrow\rangle \otimes\left|\mathrm{p}_{+}^{z}\right\rangle+\mathrm{b}|\downarrow\rangle \otimes\left|\mathrm{p}_{-}^{z}\right\rangle .(3)
$$

This situation is just a case of the measurement problem. Plus, one could also change the basis for the apparatus and realise that the formalism does not specify which observable is being measured-the preferred basis problem, see (Zurek 1982, p. 1865). At this point the environment enters.

Decoherence conceives of the composite system SA as an open quantum system that interacts at $t=0$ with a previously uncorrelated environment $E$. The environment is a large number $\mathrm{N}$ of $1 / 2$-spin systems described by bases $\left\{\left|u_{+}\right\rangle_{k},\left|u_{-}\right\rangle_{k}\right\}_{k \text { in }[1,2,3, \ldots, N]}$, so that $\left|u_{+}\right\rangle_{k}$ and $\left|u_{-}\right\rangle_{k}$ are the eigenstates of

mechanics in merely instrumental terms, and it is entirely legitimate for the realist to expect more than that.

${ }^{8}$ It is a matter of debate 'how much' metaphysics a realist interpretation of quantum mechanics should. At this stage I assume the traditional view that realism involves claims about the way the world is. 
$S_{z}^{E, k}$, the component in $z$ of the $k$ th spin of $E$. The initial state of $S A$ at $\mathrm{t}=0$ is $\left|\Psi_{\mathrm{f}}\right\rangle=\mathrm{a}|\uparrow\rangle \otimes\left|\mathrm{p}_{+}^{z}\right\rangle$, which will be now called the 'pre-measurement' state. Therefore, the new initial state $|\chi(0)\rangle$ of the composite system SAE that now includes the environment is

$$
|\chi(0)>=| \Psi_{\mathrm{i}}>\bigotimes_{\mathrm{k}=1}^{\mathrm{N}}\left(\alpha_{\mathrm{k}}\left|\mathrm{u}_{+}>+\beta_{\mathrm{k}}\right| \mathrm{u}_{-}>\right),(4)
$$

where $\alpha_{k}, \beta_{k} \in C$ and $\left|\alpha_{k}\right|^{2}+\left|\beta_{k}\right|^{2}=1$. Now, in terms of the interactions in the system SAE it is reasonable to only consider the interaction term between the apparatus $A$ and each $k$ th element of the environment $E$, which is given by

$$
\mathrm{H}_{\mathrm{k}}^{\mathrm{AE}}=\mathrm{g}_{\mathrm{k}}\left(\left|\mathrm{p}_{+}^{z}><\mathrm{p}_{+}^{z}\right|-\left|\mathrm{p}_{-}^{z}>\left\langle\mathrm{p}_{-}^{z}\right|\right) \otimes\left|\mathrm{u}_{+}><\mathrm{u}_{-}\right|_{\mathrm{k}}=\mathrm{g}_{\mathrm{k}} \mathrm{S}_{z}^{\mathrm{A}} \otimes \mathrm{S}_{z}^{\mathrm{E}, \mathrm{k}}\right.
$$

with analogous meaning for $g_{k}$ as a coupling constant in the interaction in eq. (1). Therefore, the total hamiltonian for the composite system SAE includes the interaction between the apparatus and each $k$ th element of the environment, and it is

$$
\mathrm{H}^{\mathrm{AE}}=\sum_{\mathrm{k}} \mathrm{H}_{\mathrm{k}}^{\mathrm{AE}} \cdot(6)
$$

With this Zurek (1982, p. 1865) aims at showing that this interaction hamiltonian will preclude the apparatus $S$ being in a superposition state that includes states of the apparatus in 'macroscopically' different states. Obtaining that would mean having a solution for the measurement problem and the preferred basis problem. The steps the Zurek takes to show that begin by considering the time evolution of the initial state $\mid \chi(0)>$ in eq. (4) at $\mathrm{t}=0$ to time $\mathrm{t}$ via the hamiltonian $\mathrm{H}^{\mathrm{AE}}$ from eq. (6). That calculation obtains state (considering $\hbar=1$ )

$$
\begin{gathered}
|\chi(\mathrm{t})>=\mathrm{a}| \mathrm{s}_{+}>\otimes_{\mathrm{k}}\left(\alpha_{\mathrm{k}} \exp \left(\mathrm{ig}_{\mathrm{k}} \mathrm{t}\right)\left|\mathrm{u}_{+}\right\rangle_{\mathrm{k}}+\beta_{\mathrm{k}} \exp \left(-\mathrm{ig}_{\mathrm{k}} \mathrm{t}\right) \mid \mathrm{u}_{-}>{ }_{\mathrm{k}}\right)+ \\
\mathrm{b} \mid \mathrm{s}_{-}>\otimes_{\mathrm{k}}\left(\alpha_{\mathrm{k}} \exp \left(-\mathrm{ig}_{\mathrm{k}} \mathrm{t}\right)\left|\mathrm{u}_{+}>_{\mathrm{k}}+\beta_{\mathrm{k}} \exp \left(\mathrm{ig}_{\mathrm{k}} \mathrm{t}\right)\right| \mathrm{u}_{-}>_{\mathrm{k}}\right)(7)
\end{gathered}
$$

using the notation $\left|s_{+}\right\rangle=|\uparrow\rangle \otimes\left|p_{+}^{z}\right\rangle$ and $\left|s_{-}\right\rangle=|\downarrow\rangle \otimes\left|p_{-}^{z}\right\rangle$. Now, for notation purposes, consider the following states, ${ }^{9}$

$$
\begin{gathered}
\left|\varepsilon_{+}(\mathrm{t})\right\rangle=\alpha_{\mathrm{k}} \exp \left(\mathrm{ig}_{\mathrm{k}} \mathrm{t}\right)\left|\mathrm{u}_{+}\right\rangle_{\mathrm{k}}+\beta_{\mathrm{k}} \exp \left(-\mathrm{ig}_{\mathrm{k}} \mathrm{t}\right)\left|\mathrm{u}_{-}\right\rangle_{\mathrm{k}} . \\
\left|\varepsilon_{-}(\mathrm{t})\right\rangle=\left|\varepsilon_{+}(-\mathrm{t})\right\rangle \text {. (9) }
\end{gathered}
$$

which help us to rewrite $\mid \chi(t)>$ from eq. (7) as

$$
|\chi(\mathrm{t})>=\mathrm{a}| \mathrm{s}_{+}>\otimes\left|\varepsilon_{+}(\mathrm{t})>+\mathrm{b}\right| \mathrm{s}_{-}>\otimes \mid \varepsilon_{-}(\mathrm{t})>.(10)
$$

Now let us take the statistical operator for the entire system SAE in the pure state $|\chi(\mathrm{t})\rangle$

$$
\begin{gathered}
\rho^{\mathrm{SAE}}(\mathrm{t})=|\chi(\mathrm{t})><\chi(\mathrm{t})|(11) \\
\rho^{\mathrm{SAE}}(\mathrm{t})=|\mathrm{a}|^{2}\left|\mathrm{~s}_{+}><\mathrm{s}_{+}\right| \otimes\left|\varepsilon_{+}(\mathrm{t})><\varepsilon_{+}(\mathrm{t})\right|+\mathrm{ab}^{*}\left|\mathrm{~s}_{+}><\mathrm{s}_{-}\right| \otimes\left|\varepsilon_{+}(\mathrm{t})\right\rangle\left\langle\varepsilon_{-}(\mathrm{t})\left|+\mathrm{ba}^{*}\right| \mathrm{s}_{-}><\mathrm{s}_{+}\right| \otimes \\
\left|\varepsilon_{-}(\mathrm{t})><\varepsilon_{+}(\mathrm{t})\right|+|\mathrm{b}|^{2}\left|\mathrm{~s}_{-}><\mathrm{s}_{-}\right| \otimes \mid \varepsilon_{-}(\mathrm{t})>\left\langle\varepsilon_{-}(\mathrm{t})\right| .(12)
\end{gathered}
$$

The cross-terms, the off-diagonal terms in the matrix of eq. (12) preclude the interpretation of the pure state of the total system SAE as a "classical" state. That is, the state $\rho^{\mathrm{SAE}}(\mathrm{t})$ has coherences. The coherence of the overall state $\rho^{\mathrm{SAE}}(\mathrm{t})$ is maintained always and cannot change through a unitarian evolution (which is the type of evolution that we assumed throughout, given by the Schrödinger equation). Instead, what the process of decoherence obtains is a 'redistribution' of such coherences.

Now, when the system is composite, one can obtain a description of one of the subsystems by tracing out the degrees of freedom of the rest of the total system. In this case, we trace out the degrees of freedom of the environment $E$ in order to obtain the statistical operator of the subsystem SA:

${ }^{9}$ Following the standard account presented in Zurek (1982, p. 1864) and Zurek (2003, p. 730). 


$$
\begin{gathered}
\rho^{\mathrm{SA}}(\mathrm{t})=\operatorname{Tr}_{\mathrm{E}}(|\chi(\mathrm{t})\rangle\langle\chi(\mathrm{t})|) \\
\rho^{\mathrm{SA}}(\mathrm{t})=|\mathrm{a}|^{2}\left|\mathrm{~s}_{+}\right\rangle\left\langle\mathrm{s}_{+}\right|+\mathrm{ab}^{*}\left\langle\varepsilon_{-}(\mathrm{t})\left|\varepsilon_{+}(\mathrm{t})>\right| \mathrm{s}_{+}><\mathrm{s}_{-}\left|+\mathrm{ba}^{*}<\varepsilon_{-}(\mathrm{t})\right| \varepsilon_{+}(\mathrm{t})>\right| \mathrm{s}_{-}>\left\langle\left.\mathrm{s}_{+}|+| \mathrm{b}\right|^{2}\left|\mathrm{~s}_{-}><\mathrm{s}_{-}\right| .\right.
\end{gathered}
$$

The achievement of decoherence through the monitoring effect of the environment $E$ on the subsystem composed by the system and apparatus SA is to "damp out" the correlations, the off-diagonal terms in eq. (13).

Let us note the correlation amplitude $\left\langle\varepsilon_{+}(\mathrm{t}) \mid \mathcal{E}_{-}(\mathrm{t})\right\rangle={ }_{z}(\mathrm{t})$. Zurek (1982, p. 1866) obtains-see also (Schlosshauer 2005, p. 1277)-that the average for large times of $|z(t)|^{2}$ becomes significantly small when taking the limit $\mathrm{N} \rightarrow \infty$ :

$$
\left\langle|z(\mathrm{t})|^{2}\right\rangle_{\mathrm{t} \rightarrow \infty} \approx 2^{-\mathrm{N}} \prod_{\mathrm{k}=1}^{\mathrm{N}}\left[1+\left(\left|\alpha_{\mathrm{k}}\right|^{2}-\left|\beta_{\mathrm{k}}\right|^{2}\right)^{2}\right] \rightarrow_{\mathrm{N} \rightarrow \infty} \mathrm{O}
$$

This last formula shows that the correlation amplitude $z(t)$ which is 1 at $\mathrm{t}=0$, decreases exponentially fast with the size $N$ of the environment coupled with the apparatus and is zero when $t=\infty$.

As Schlosshauer (2005, pp. 1277-1279) discusses, the damping happens in a really short time. Even microscopic systems are rapidly decohered by the interaction with the environment, the thermal radiation for instance. The time within which $|z(t)| \rightarrow 0$ is of a much shorter scale than any practical observation could resolve. In addition, with a similar model of decoherence that the one discussed here, Joos and Zeh (1985) analyse the case of a dust grain floating in the air at room temperature. Defining a coherence length as a distance beyond which no interference should be shown, they find that it is the same that its de Broglie wavelength $\lambda^{-14} \mathrm{~cm}$, for a dust speck of radius $10^{-5} \mathrm{~cm}$. The time in which the coherence is delocalised for a system in a minimal environment is surprisingly quick. Further calculations are shown in (Bacciagaluppi 2000) and see (Bacciagaluppi 2016).

Another case where decoherence succeeds with establishing the adequacy of quantum predictions is in the dynamics of the moon Hyperion (a classical system), which chaotically orbits around Jupiter. Whilst the time when classical and quantum predictions diverge from each other is around 20 years, the system is about 4 billion years old. Hence, quantum effects should have been observed in its orbit. But this contradicts the empirical fact that Hyperion looks classical. Decoherence solves this disagreement between classical and quantum description of the system, see (Zurek \& Paz 1997). Zurek and $\mathrm{Paz}$ showed that the environment secures the seemingly classical dynamic of the moon, because the quantum superpositions decohere.

The tendency of $|z(t)|$ to go to zero so quickly shows the efficacy of decoherence. Under these conditions, any observable belonging to the system + apparatus SA would have, for all practical purposes, the same mean values that would have obtained had the system SA been a described by the mixture

$$
\rho^{\prime S A}(t)=|a|^{2}\left|s_{+}><s_{+}\right|+|b|^{2}\left|s_{-}><s_{-}\right|
$$

The discussion of how this helps with the measurement problem and the problem of the preferred basis has been discussed widely. The idea is that in a typically very short time the reduced density matrix in eq. (13) including off-diagonal terms, becomes approximately diagonal

$$
\begin{aligned}
& |z(\mathrm{t})| \rightarrow_{\mathrm{N}, \mathrm{t} \rightarrow \infty} 0, \\
& \rho^{\mathrm{SA}} \rightarrow|z(\mathrm{t})| \rightarrow 0|\mathrm{a}|^{2} \mid \uparrow>\left\langle\uparrow|\otimes| \mathrm{p}_{+}^{z}>\left\langle\left.\mathrm{p}_{+}^{z}|+| \mathrm{b}\right|^{2} \mid \downarrow\right\rangle<\downarrow|\otimes| \mathrm{p}_{-}^{z}>\left\langle\mathrm{p}_{-}^{z}\right|\right. \text { (16) }
\end{aligned}
$$


where I used the previous notation $\left|s_{+}\right\rangle=|\uparrow\rangle \otimes\left|p_{+}^{z}\right\rangle$ and $\left|s_{-}\right\rangle=|\downarrow\rangle \otimes\left|p_{-}^{z}\right\rangle$. This is interpreted in the following way: although the unitary evolution does not destroy the global phase coherence, such a coherence is delocalised away from the reduced density matrix that describes the system and apparatus SA to the degrees of freedom of the environment E, see (Schlosshauer 2005,p.1276). The effect of "environment-induced decoherence" is to have arrived at approximately diagonal density matrix for SA, displaying pointer states which remain in spite of the environment, whilst their superpositions lose phase coherence and decohere, see (Zurek 2003, p. 707). In conclusion, the environment effectively "destroys" the correlation between states that correspond to different eigenvalues of $\mathrm{H}^{\mathrm{SA}}$, the environment precludes coherent superpositions.

With regards to the preferred basis problem, the effect of the environment was developed in Zurek (1981) with the so-called the environment-induced superselection. Decoherence selects the preferred pointer basis, which does not change the system-apparatus correlations despite the interaction between the apparatus and the environment, see Zurek (1981, p. 1520) and Schlosshauer (2005, pp. 12781279). The preferred observable selected due to the interaction with the environment is

$$
\mathrm{O}_{\mathrm{A}}=\lambda_{1} \mid \mathrm{p}_{+}^{z}>\left\langle\mathrm{p}_{+}^{z}\left|+\lambda_{2}\right| \mathrm{p}_{-}^{z}>\left\langle\mathrm{p}_{-}^{z}\right|,\right.
$$

where $\lambda_{1}, \lambda_{2} \in \mathrm{R}$. Zurek (1981, p. 1522) proves that the apparatus-system will retain perfect correlation in only one product basis of $S A,\left\{|\uparrow\rangle \otimes\left|p_{+}^{z}\right\rangle,|\downarrow\rangle \otimes\left|p_{-}^{z}\right\rangle\right\}$. Hence pointer basis states of the apparatus $\operatorname{are}\left\{\left|p_{+}^{z}\right\rangle,\left|p_{-}^{z}\right\rangle\right\}$. Therefore, the correlations S-system-A-apparatus between pointer states that are not eigenstates of an observable that commutes with $\mathrm{H}^{\mathrm{AE}}$ will be quickly delocalised by the monitoring effect of the environment over the apparatus, see Schlosshauer (2005).

As said before, I am interested in discussing two sources of worry from a foundational or conceptual point of view for considering decoherence within a realist framework.

\subsection{Objections to decoherence as a realist account of the quantum-classical limit}

Predictions made through the theory of decoherence have an incredible accuracy and the work of Joos, Zeh, Zurek, Paz, and many others, represent a substantial advance in physics. The empirical accuracy strongly suggests that at least something like the process of decoherence does happen in nature. However, I claim that there are serious concerns for the realist. It often happens that useful and reliable tools for making predictions in physics, are not uncontroversial for the philosopher who considers a conceptual point of view. Consider, for example, Dirac's delta function, renormalisation in quantum field theory, or even Feynman's path integral formalism. I claim that one can critically engage with the philosophy of physics and the practice of physicists, without denying the physics itself.

My criticism of the claim that decoherence is the ultimate solution to the problems of quantum mechanics and a description of the quantum-classical limit, involves two issues. Firstly, I argue that decoherence works only "for all practical purposes" and that this is problematic in a special sense that I will clarify and, secondly, that there is a potentially vicious circularity involved (Kastner 2014).

The first issue relates to the distinction between proper and improper mixtures. This ambiguity in the physical interpretation of the mathematical entity of the density operator has been known since the 1970s with d'Espagnat $(1976,1995)$ and Hellwig and Kraus (1968). ${ }^{10}$ The argumentative force of the distinction is perhaps irrelevant when conceptual issues are considered with less emphasis than pragmatic, empirical ones. Indeed, the incredibly successful applications of decoherence-based approaches do not recognise a troubling issue behind this distinction. However, the realist is concerned with foundational questions, and for her the distinction between proper and improper mixtures ought to be of crucial relevance, as I will argue. The following discussion is well-known in the philosophical literature. The bottom line is that the final state of the model of decoherence is a limit obtained from the density matrix in eq. (13), which is an improper mixture, and improper mixtures

\footnotetext{
${ }^{10}$ Interesting discussion about the history of the improper mixtures is given in Bub (1997, Sec. 8.1.) and Masillo et al. (2009).
} 
cannot be simply used to describe one of the subsystems of a composite system. Plus, it is general unclear how to interpret improper mixtures altogether.

Let us consider a state $\rho$ for an entangled bi-partite system in a finite-dimension case

$$
\rho=|\Psi\rangle\left\langle\Psi\left|=\sum_{\mathrm{ij}} \mathrm{a}_{\mathrm{i}} \mathrm{a}_{\mathrm{j}}^{*}\right| \alpha_{\mathrm{i}} \otimes \beta_{\mathrm{i}}>\left\langle\alpha_{\mathrm{j}} \otimes \beta_{\mathrm{i}}\right| .\right.
$$

Given that this state is entangled it is just not possible to consider individual subsystems with individual properties. Nevertheless, there is a trick that allows you to 'separate' the composite system in subsystems: by using improper mixtures. Say that we are only capable of performing measurements of one of the subsystems, the observables for that case will be of the type $\mathrm{O}_{\mathrm{S}} \otimes 1_{\mathrm{E}}$ and $1_{\mathrm{S}} \otimes \mathrm{O}_{\mathrm{E}}$. Then we will, at best, be able to reconstruct the density operators given by the partial traces:

$$
\begin{aligned}
& \rho_{\mathrm{S}}=\operatorname{Tr}^{\mathrm{E}}\left(|\Psi>\langle\Psi|)=\sum_{\mathrm{n}}\left|\mathrm{a}_{\mathrm{n}}\right|^{2} \mid \alpha_{\mathrm{n}}>\left\langle\alpha_{\mathrm{n}}\right|\right. \\
& \rho_{\mathrm{E}}=\operatorname{Tr}^{\mathrm{S}}(|\Psi\rangle\langle\Psi|)=\sum_{\mathrm{n}}\left|\mathrm{b}_{\mathrm{n}}\right|^{2} \mid \beta_{\mathrm{n}}>\left\langle\beta_{\mathrm{n}}\right|
\end{aligned}
$$

However, because $\rho$ in eq. (18) is entangled, the product of the matrices in eqs. (19) and (20) obviously does not obtain $\rho$ but $\rho$

$$
\rho^{\prime}=\rho_{\mathrm{S}} \otimes \rho_{\mathrm{E}} \cdot(21)
$$

Let me insist with the qualitative difference between these two matrix operators. In eq. (21) we have $\rho$ ', which is a separable state that is not a projection operator and it cannot represent a pure state because $\rho^{\prime 2} \neq \rho^{\prime}$. By contrast, $\rho$ from eq. (18) describes an entangled composite system in a pure state. The nature of the physical system cannot change as a result of a mathematical operation such as taking a partial trace, obviously. Now, can we re-construct $\rho$ by performing measurements on the subsystems only? No we cannot. Instead, only $\rho$ ' can be known from such a type of measurements. In order to obtain $\rho$ one would require to know the correlations between the subsystems, which by hypothesis are not available. The coherences that make $\rho^{\prime} \neq \rho$ are coded in the phases of the coefficients $a_{i}$ in eqs. (19) and (20). And precisely those phases are lost when tracing out the degrees of freedom of one of the subsystems, given that the matrixes in eqs. (19) and (20) include the absolute value of the coefficients. The correlations cannot be observed or detected by measuring on the partial systems only. The correlations can only be measured by measuring on the entire composite system, see (d'Espagnat 1995, Ch. 7) and (Primas 1981, p. 144). Therefore, one cannot simply claim that the density operators in eqs. (19) and (20) describe the subsystems $S$ and $E$, respectively.

Those two improper mixtures describe the subsystems only in a restricted manner (as just discussed). In relation to decoherence, the final state of the system + apparatus + environment SAE in eq. (11) is a pure entangled state. Then, given that the state for the subsystem SA in eq. (13) was obtained by tracing out the degrees of freedom of the environment, the resulting state is an improper mixture. One ought not to confuse separable systems with entangled systems, one ought not to take that the improper mixture describes the subsystem simpliciter. As discussed above, by tracing out of degrees of freedom there is a loss of phase coherences (regardless of whether the improper mixture itself is diagonal, approximately diagonal, or non-diagonal). If the realist takes the formalism seriously (and she should), the entangled nature of the system is still there, even if one traces out degrees of freedom of the environment.

Let us discuss the consequences of the interpretation of proper mixtures. Following d'Espagnat (1976, p. 44), a proper mixture is a statistical mixture of systems in pure states obtained from considering a mixture of $N_{1}$ systems in pure state $\left|\phi_{1}\right\rangle, N_{2}$ systems in pure state $\left|\phi_{2}\right\rangle$, and so on, with the condition that $\sum_{\alpha} \mathrm{N}_{\alpha}=\mathrm{N}$. A proper mixture is described by the statistical operator 


$$
\rho_{\mathrm{P}}=\sum_{\alpha} \frac{\mathrm{N}_{\alpha}}{\mathrm{N}} \mid \phi_{1}>\left\langle\phi_{1}\right| .
$$

This operator is mathematically equivalent to the improper mixture obtained by tracing out degrees of freedom of a larger system in eqs. (19) and (20). Presented with a statistical operator only, one cannot know whether it is a proper or an improper mixture, unless further information is supplied. Essentially, all one could know from the mathematical entity, is whether the state is pure (if the statistical operator is a projection operator) or a mixture (if it is not a projection). However, despite their mathematical identical appearance, the physical interpretation of proper and improper mixtures is significantly different. The statistical mixture in the system represented by $\rho_{P}$ refers to ignorance of the observer over a determined state of affairs. This is similar to the classical ensemble of many identical systems: we know that the system is an element of the ensemble, but we ignore-we cannot say-which one with our limited knowledge. We also assume that all the properties of the classical system are determined, but we do not know their values. Therefore, the probabilistic character of the statements made here relate to our ignorance of the state of the system. In the case of the proper mixture we know that the system is in a pure state, but we do not know which one.

By contrast, the probabilities involved in the improper case cannot be interpreted by ignorance in the way just described. This is widely known in the philosophical literature, e.g. d'Espagnat (1976), Landsman (2007, p. 516), Holik, de Ronde and Christiaens (2013), Fortin and Lombardi (2014), Ladyman and Ross (2007, p. 177). In turn, the physics literature typically neglects the distinction altogether. The standard textbooks do not teach physicists this crucial distinction proper/improper, thus the literature misinterprets the states obtained from tracing out degrees of freedom. ${ }^{11}$ However, the philosopher has a critical role in accounting not only for the practice of the scientist, but for the conceptual implications of the theory as well. Crucially, the mistake in the standard interpretation of the model of decoherence is the assumption that the subsystem is really described by a proper mixture $\rho=|\mathrm{a}|^{2}|\uparrow\rangle\left\langle\uparrow|\otimes| \mathrm{p}_{+}^{z}\right\rangle\left\langle\left.\mathrm{p}_{+}^{z}|+| \mathrm{b}\right|^{2} \mid \downarrow\right\rangle\left\langle\downarrow|\otimes| \mathrm{p}_{-}^{z}\right\rangle\left\langle\mathrm{p}_{-}^{z}\right|$, which the model of decoherence obtains in the limit when $\mathrm{t} \rightarrow \infty$ and $\mathrm{N} \rightarrow \infty$, and $\rho^{\mathrm{SA}}(\mathrm{t})=\operatorname{Tr}_{\mathrm{E}}(|\chi(\mathrm{t})><\chi(\mathrm{t})|) \rightarrow \rho$ as the coherences become delocalised given that the correlation $|z(t)|=\mid\left\langle\varepsilon_{+}(t)\left|\varepsilon_{-}(t)>\right| \rightarrow 0\right.$, see eqs. (13), (14) and (16). However, because of the quantum entanglement between the apparatus $A$ and environment $E$, one cannot presuppose that the subsystem SA has an individual state. Paradoxically, this misconception does not present an obstacle to the physicists to make accurate predictions which are successful 'for all practical purposes' (FAPP) only.

In order to avoid having to interpret the improper mixtures and include the FAPP-style success of the standard models of decoherence, the realist requires further arguments. Those arguments could indicate that quantum mechanics is only approximately true in the sense that there is a more fundamental theory that is closer to the truth. However, this argument would be dependent on more fundamental physics, which is yet to be developed. Furthermore, if the realist argued in this way in order to justify the FAPP interpretation of the quantum formalism, she would be utilising, at a crucial level, arguments that rely on conceptions of intertheory relations. As discussed above, a proper methodology would firstly interpret the theory realistically and then establish a relationship with other theories and their real content.

In addition to the conceptual difficulties around the difference between proper and improper mixtures, there is a second concern for the realist whose view depends relies on decoherence, which has been put forward by Kastner (2014). Her argument targets the role that decoherence plays in the many worlds interpretation. However, all that is required for the argument to work is that only unitarian evolution is assumed in quantum mechanics. Hence, her criticism can be extended to a criticism of any non-collapse interpretation. Essentially, she notes that the model of decoherence that

\footnotetext{
${ }^{11}$ Current literature in foundations of physics fails to understand the meaning of this difference. For instance, Nielsen and Chuang (2010, p. 106) tell us that partial traces give us a "state about which we apparently do not have maximal knowledge". This is misleading for partial traces obtain an improper mixtures that cannot be interpreted in terms of ignorance of an actual state of affairs. Such an assertion is true for proper mixtures, see d'Espagnat $(1976,1995)$.
} 
intends to explain the vanishing of the off-diagonal terms in eq. (13) through the limit $|z| \rightarrow 0$ in eq. (14), is circular and invalid. This is because it is implicitly assumed that the apparatus has decohered from the beginning. In her words:

\begin{abstract}
macroscopic classicality only 'emerges' in [the many world interpretations] picture because a classical, non-quantum-correlated environment was illegitimately put in by hand from the beginning. Without that unjustified presupposition, there would be no vanishing of the off-diagonal terms and therefore no apparent diagonalization of the system's reduced density matrix that could support even an approximate, 'FAPP' mixed state interpretation. (Kastner 2014, p. 57)
\end{abstract}

The underlying argumentative force depends too on identifying the distinction between improper mixtures from proper mixtures, and that decoherence obtains improper mixtures.

Consider as Bacciagaluppi does, that according to the conception of open systems in the model of decoherence, "everything is in interaction with everything else, everything is generically entangled with everything else" (Bacciagaluppi 2016, Sect. 2). That is, everything is entangled with everything else, and there are no pure separable states, strictly speaking. However, as Kastner (2014) points out the theory of decoherence considers that at the instant of time when interaction apparatus-environment is set on, the state of the entire system (system and apparatus SA, and the environment E) is the pure state $|\div(0)\rangle$ in eq. (4). $\left|\Psi_{\mathrm{f}}\right\rangle$ is product state of the pre-measurement state of the subsystem $S A,\left|\Psi_{\mathrm{f}}\right\rangle$ and the state of the environment $E, \bigotimes_{k} \alpha_{k}\left|u_{+}\right\rangle_{k}+\beta_{k}\left|u_{-}\right\rangle_{k}$. Hence, $\mid \chi(0)>$ is a product state of these two.

However, if the hypothesis of decoherence is that quantum systems are open and what Bacciagaluppi says is true, then the components of the system must have already decohered completely, previous to that instant $t=0$. On the other hand, the model of decoherence does not obtain a pure state as a final state, but improper mixtures. Even if one ignores the distinction proper/improper, a mixed state is not a pure state. Therefore, following Bacciagaluppi and Kastner, decoherence uses a conclusion within premises. In a nutshell: if the state before the interaction with the environment is a product state, where did that pure state come from?

With this I conclude the discussion of decoherence. If I am right and decoherence does play a role in the account of the quantum-classical limit and it is not a sound account of it, then the realist has to find another way out. In the next section I will offer three alternatives for how to do so.

\title{
4. Alternatives to the received account of the quantum-classical relation
}

The theoretical devices that attempt to address the quantum-classical relation have, as I have argued, conceptual limitations. The most these methods can achieve is to recover something that resembles classical physics, in a limited sense. Now, suppose that one accepted the explanation of the appearance of the classicalities, and the solution to the measurement problem and the preferred basis problem, through decoherence. Then, what realist account is there for the cases from which no classicalities appear? What about cases where the coherence is retained, such as in quantum computation? There, decoherence effects are precisely what the physicist wants to avoid and really complex experimental techniques, such as ion traps, are used in order to provide a long coherence time that would allow developments in the field of quantum computation, see (Cirac \& Zoller 1995). Furthermore, there are cases where there is a coherent superposition which has direct experimental results, such as any quantum experiment. In relation to decoherence as an answer to how quantum mechanics reduces to classical mechanics, there is experimental and theoretical evidence of effects that are purely quantum and with direct expression in the 'macroscopic' realm, see (Brezger et al. 2002, Kovachy et al. 2015) and (Landsman 2007, p. 418, and references therein).

The arguments in previous sections intended to show that the traditional account of the quantumclassical limit has shortcomings and the realist cannot succeed in providing a smooth account of the appearance of classicalities from the quantum. Moreover, even if she did provide such an account, she 
would still need a realist interpretation of quantum mechanics including those cases where no classicalities emerge.

Now, the recognition of the overall limitations of the traditional account of the quantum-classical relation in light of the limitations of the formal theoretical devices developed in physics, is shared by many, see Bokulich (2008a, Ch. 1), Landsman (2007). The distinction I would like to make is over how to react. What does these limitations indicate and how to fix them? My view is that the debates on the difficulties around the quantum-classical limit involve mainly three positive alternatives, arising from modifying one of the three elements in the very debate.

The problem can be visualised by considering a triangle that has at its vertices the type of intertheory relation, the philosophical account of it, and the physical account. This has the advantage of suggesting that changes might come in by replacing one or more vertices of the triangle. I illustrate these elements with the triangle in Figure 1, where I considered the standard view.

As discussed above, the standard view takes it that quantum mechanics is more fundamental than classical mechanics and thus the former has to show the appearance of the latter. This is a type of conception of intertheory relation (the top vertex). Yet, that intertheory relation can be realised in various ways: reduction, emergence and even forms of pluralism, among others. Those views appear in the bottom left vertex, where the standard one is, I take it, theory reduction. More specifically, the form of theory reduction that is considered standard for physics is Nickles' 'physicists' reduction, see (Nickles 1973). Finally, the way in which this type of intertheory relation, realised in a particular philosophical account, is actually related to physics, appears on the right vertex. Various theoretical devices are meant to be the realisation of the other two vertices. In particular, the mathematical limits, Ehrenfest theorem, Moyal brackets, and decoherence, are well-known. In the previous section I discussed in detail how decoherence is meant to account for the relation quantum-classical.

Now I will mention relevant and recent research focused on each of the three options, although for space reasons I will not critically engage with them. One could claim that the standard account of the quantum-classical limit is insufficient because of the reasons discussed above and, crucially, because there are relevant cases of physics that do not fit at all with such a framework. Thereby this option challenges specifically the top vertex of the triangle in Figure 1, and modifies the type of intertheory relation. Bokulich (2008a, 2008b, 2012) takes such an approach. She advances a novel intertheory relationship that goes beyond the traditional vertical hierarchy emphasised by theory reduction (and also some forms of pluralism). Her argument focuses on indicating the difficulties of the standard view to accommodate semiclassical phenomena. Bokulich's view, interstructuralism, is designed to accommodate physical cases that mix elements from classical mechanics and from quantum mechanics.

More specifically, interstructuralism considers relevant physical systems in the mesoscopic scalesuch as Rydberg atoms in strong uniform external magnetic fields-that cannot be explained by quantum mechanics alone, let alone fit in the schemes of the traditional account of the quantumclassical limit. Such a system lies in the empirical region between the micro-quantum mechanics and the macro-classical mechanics. Therefore, the alternative here is to articulate a novel conception of intertheory relation.

Secondly, one could modify the left vertex of the triangle in Figure 1. This would maintain theory reduction as the appropriate intertheory relation but change the form of such reduction to better capture the relevant physics of the quantum-classical relation. The advocate of this view could claim that decoherence-seen as the culmination of the account of the quantum-classical limit-is the appropriate device to account for the reduction relation. Yet they will criticise and modify the traditional philosophical views on theory reduction. In this line, Rosaler (2016) puts forward a one-sizefits-all account of reduction designed to work equally well for all the different interpretations of quantum mechanics and is based on decoherence as a template for such reduction. ${ }^{12}$ His reduction relationship is based on decoherence and it is meant to be an interpretation-neutral approach.

\footnotetext{
${ }^{12}$ Rosaler considers the different interpretations to be different physical theories "since they differ in the accounts of physical reality (in particular, the laws and ontology) that they take to underwrite the success of the quantum formalism" (Rosaler 2016, p. 55). Is there a reduction relationship between these different theories? Will the reduction be the same as the one he claims to exist between the various
} 


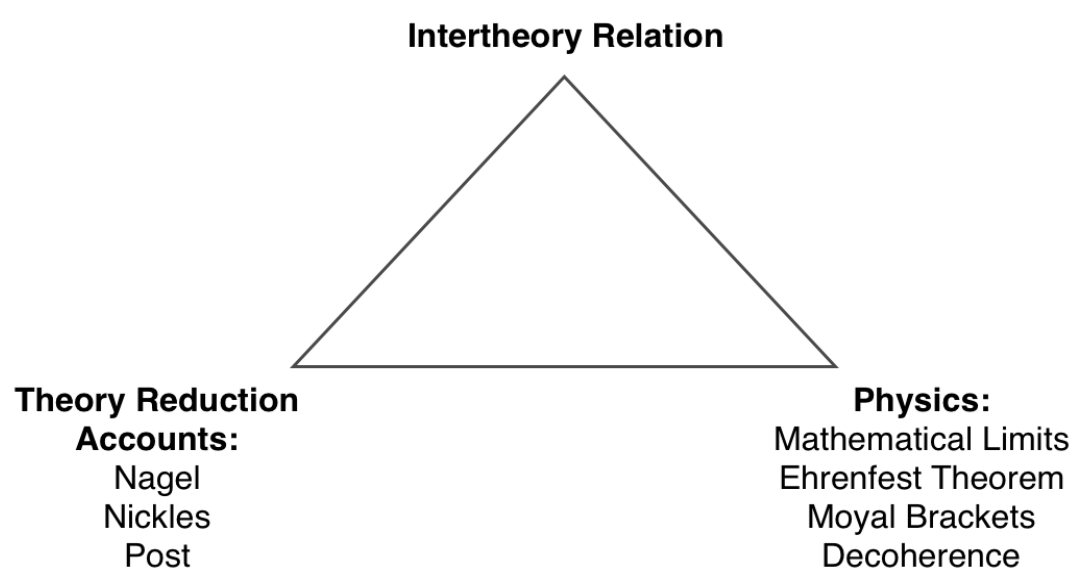

Figure 1: Triangle of the quantum-classical limit. The possible strategies to advance a novel view are initiated by targeting one of the three vertices shown by modifying, improving, criticising them.

Finally, one could focus on the bottom right vertex of the triangle in Figure 1 and challenge the consideration of the physics involved. A reaction to the difficulties of the standard philosophical account of the quantum-classical limit based on theory reduction and the physics of decoherence and other devices, could be to modify the physical account of the quantum-classical limit itself. Relevant work is conducted by Kastner (2014), which I comment on very briefly. Although she does not directly engage in the debate on theory reduction, we can point out that in the face of controversies around the process of decoherence to account for the appearance of classicalities, Kastner argues that the empirical fact that we observe decoherence is not derivable from a unitary-only dynamics. Hence, she suggests that the relativistic field picture may be promising as a solution to this. She argues that in order to tackle the questions arising within non-relativistic quantum mechanics, one has to move on to a relativistic approach. Her view considers that the energy-momentum (in the 4-momentum form) basis is more fundamental than position, and thus takes energy/momentum transfers as primary and the spacetime phenomena as secondary and emergent, supervening on the E, p transfer. These are the central elements for her ontology of Possibilist Transactional Interpretation, see Kastner (2012).

My proposal is to insist that the central question is the interpretation question: what is a quantum system? This does not have a clear answer and this is what the realist interpretation of quantum mechanics should focus on. The emphasis on decoherence and the account of the quantum-classical limit within the debates on the realist interpretation of quantum mechanics evidence that today we seem to be more concerned about the intertheory relation than in the actual question of relevance. The role of the intertheory relations is overrated, and I persuade the realist to focus on quantum mechanics alone, and not in relation to classical mechanics. An approach that interprets quantum mechanics alone will take the problem to establish a relationship between the formalism and the world (not between the formalism and the classical world). Such an approach is already being undertaken, see (Ronde 2017).

\section{References}

Bacciagaluppi, G. (2000), "Delocalized Properties in the Modal Interpretation of a Continuous Model of Decoherence", Foundations of Physics 30(9): 1431-1444. 
Bacciagaluppi, G. (2013), "Measurement and Classical Regime in Quantum Mechanics”, in Batterman, R. (ed.), The Oxford Handbook of Philosophy of Physics, New York: Oxford University Press, pp. 416-459.

Bacciagaluppi, G. (2016), “The Role of Decoherence in Quantum Mechanics”, in Zalta, E. N. (ed.), The Stanford Encyclopedia of Philosophy (Fall 2016 Edition). URL = http://plato.stanford.edu/archives/fall2016/entries/qmdecoherence/.

Bokulich, A. (2008a), Reexamining the Quantum-Classical Relation: Beyond Reductionism and Pluralism, Cambridge: Cambridge University Press.

Bokulich, A. (2008b), "Can Classical Structures Explain Quantum Phenomena?", British Journal for the Philosophy of Science 59(2): 217-235.

Bokulich, A. (2012), "Distinguishing Explanatory from Nonexplanatory Fictions”, Philosophy of Science 79(5): 725-737.

Brezger, B., Hackermaler, L., Uttenthaler, S., Petschinka, J., Arndt, M. and A. Zeilinger (2002), "Matter-Wave Interferometer for Large Molecules”, Physical Review Letters 88(10): 100404.

Bub, J. (1997), Interpreting the Quantum World, Cambridge: Cambridge University Press.

Butterfield, J. (2011), "Emergence, Reduction and Supervenience: A Varied Landscape”, Foundations of Physics 41(6): 920-959.

Cirac, J. I. and P. Zoller (1995), "Quantum Computations with Cold Trapped Ions", Physical Review Letters 74: 40914094.

de Ronde, C. (2017), "Representational Realism, Closed Theories and the Quantum to Classical Limit", in Kastner, R. E., Jeknic-Dugic, J. and G. Jaroszkiewicz (eds.), Quantum Structural Studies, Singapore: World Scientific, pp. 105135.

d'Espagnat, B. (1976), Conceptual Foundations of Quantum Mechanics, Reading, MA: Benjamin.

d'Espagnat, B. (1995), Veiled Reality, Reading-MA: Addison-Wesley Publishers.

Fletcher, S. (2014), Similarity and Spacetime: Studies in Intertheoretic Reduction and Physical Significance, PhD thesis, University of California: Irvine.

Fortin, S. and O. Lombardi (2014), "Partial Traces in Decoherence and in Interpretation: What do Reduced States Refer to?", Foundations of Physics 44(4): 426-446.

French, S. and K. McKenzie (2012), "Thinking Outside the Toolbox: Towards a more Productive Engagement Between Metaphysics and Philosophy of Physics", European Journal of Analytic Philosophy 8(1): 42-59.

Hellwig, K. E. and K. Kraus (1968), "Pure Operations and Measurements", Communications in Mathematical Physics 11(3): 214-220.

Holik, F., de Ronde, C. and W. Christiaens (2013), "Un nuevo esquema conceptual para la interpretación de las mezclas impropias en mecánica cuántica”, Scientiae Studia 11: 101-118.

Joos, E. (1996), "Introduction”, in Giulini, D., Joos, E., Kiefer, C., Kupsch, J., Stamatescu, I. O. and H. D. Zeh (eds.), Decoherence and the Appearance of a Classical World in Quantum Theory, Berlin, London: Springer, pp. 1-4.

Joos, E. and H. D. Zeh (1985), "The Emergence of Classical Properties through Interaction with the Environment", Zeitschrift für Physik B Condensed Matter 59(2): 223-243.

Kastner, R. E. (2012), The Transactional Interpretation of Quantum Mechanics, Cambridge: Cambridge University Press.

Kastner, R. E. (2014), "Einselection of Pointer Observables: The new H-theorem?" Studies in History and Philosophy of Science Part B: Studies in History and Philosophy of Modern Physics 48: 56-58.

Kovachy, T., Asenbaum, P., Overstreet, C., Donnelly, C. A., Dickerson, S. M., Sugarbaker, A., Hogan, J. M. and M. A. Kasevich (2015), "Quantum Superposition at the Half-Metre Scale", Nature 528(7583): 530-533.

Ladyman, J. and D. Ross (2007), Everything Must Go: Metaphysics Naturalized, Oxford: Oxford University Press.

Landsman, N. P. (2007), "Between Classical and Quantum", in Butterfield, J. and J. Earman (eds.), Handbook of the Philosophy of Science: Philosophy of Physics, Amsterdam; London: Elsevier, pp. 417-554.

Masillo, F., Scolarici, G. and S. Sozzo (2009), "Proper Versus Improper Mixtures: Toward a Quaternionic Quantum Mechanics", Theoretical and Mathematical Physics 160(1): 1006-1013. 
Nickles, T. (1973), “Two Concepts of Intertheoretic Reduction”, The Journal of Philosophy 70(7): 181-201.

Nielsen, M. A. and I. L. Chuang (2010), Quantum Computation and Quantum Information, Cambridge: Cambridge University Press, 10th Anniversary Edition.

Primas, H. (1981), Chemistry, Quantum Mechanics and Reductionism: Perspectives in Theoretical Chemistry, Lecture Notes in Chemistry 24, Berlin: Springer Berlin Heidelberg.

Rosaler, J. (2016), "Interpretation Neutrality in the Classical Domain of Quantum Theory", Studies in History and Philosophy of Science Part B: Studies in History and Philosophy of Modern Physics 53:54-72.

Schlosshauer, M. (2005), "Decoherence, the Measurement Problem, and Interpretations of Quantum Mechanics", Reviews of Modern Physics 76: 1267-1305.

Schlosshauer, M. (2007), Decoherence and the Quantum-to-Classical Transition, Berlin/Heidelberg: Springer.

Wallace, D. (2016), Conversation with Nahuel Sznajderhaus, 20th May.

Zeh, H. D. (1970), "On the Interpretation of Measurement in Quantum Theory", Foundations of Physics 1(1): 69-76.

Zeh, H. D. (1996), "The Program of Decoherence: Ideas and Concepts", in Giulini, D., Joos, E., Kiefer, C., Kupsch, J., Stamatescu, I. O. and H. D. Zeh (Eds.), Decoherence and the Appearance of a Classical World in Quantum Theory, Berlin/London: Springer, pp. 5-34.

Zurek, W. and J. P. Paz (1997), "Why We don't Need Quantum Planetary Dynamics: Decoherence and the Correspondence Principle for Chaotic Systems", in Hu, B. and D. Feng (eds.), Quantum Classical Correspondence: Proceedings of the 4th Drexel Symposium on Quantum Nonintegrability, Drexel University, September 8-11, 1994, Cambridge, MA: International Press, pp. 367-79.

Zurek, W. H. (1981), "Pointer Basis of Quantum Apparatus: Into what Mixture does the Wave Packet Collapse?", Physical Review D 24: 1516-1525.

Zurek, W. H. (1982), “Environment-induced Superselection Rules”, Physical Review D 26: 1862-1880.

Zurek, W. H. (2003), "Decoherence, Einselection, and the Quantum Origins of the Classical", Reviews of Modern Physics 75: 715-775. 